\title{
Idiosyncratic cleavage and ligation activity of individual hammerhead ribozymes and core sequence variants thereof
}

\author{
Rita Przybilski and Christian Hammann* \\ AG Molecular Interactions, Department of Genetics, \\ University of Kassel, Heinrich-Plett-Str. 40, D-34132 \\ Kassel, Germany \\ ${ }^{*}$ Corresponding author \\ e-mail: c.hammann@uni-kassel.de
}

\begin{abstract}
The hammerhead ribozyme is a small RNA endonuclease found in sub-viral plant pathogens, in transcripts from certain animal satellite DNAs and encoded at distinct loci of Arabidopsis thaliana. Kinetic analyses of tertiary stabilised ribozymes from peach latent mosaic viroid (PLMVd), Schistosoma mansoni and $A$. thaliana revealed a ten-fold difference in cleavage rates. Core nucleotide variations affected cleavage reactions least in the $A$. thaliana ribozyme, and most in the $S$. mansoni ribozyme. The reverse ligation reaction was catalysed efficiently by the PLMVd and $A$. thaliana ribozymes. The different behaviour of the individual hammerhead ribozymes is discussed in terms of structure and function.
\end{abstract}

Keywords: catalytic RNA; divalent metal ions; RNA structure; satellite DNA; viroid.

Because of its relatively simple architecture (Figure 1A), the hammerhead ribozyme is among the most widely studied of the small nucleolytic ribozymes (Lilley, 2005). For some considerable time, a minimal version of the ribozyme (Uhlenbeck, 1987) has been studied (Figure $1 \mathrm{~B})$, which is characterised by three helical stems that surround a catalytic core of 11 conserved nucleotides. The core nucleotide conservation was determined in an extensive mutagenesis study, in which every nucleotide was exchanged for any other (Ruffner et al., 1990). Except for nucleotide 7 in the core region (Figure 1A), no other nucleotide could be exchanged without reducing the relative ribozyme activity to $6 \%$ or less, up to full inactivity.

In 2003, the groups of Khvorova and Flores showed independently that in natural hammerhead sequences, tertiary interactions outside the core region allow ribozyme activity under physiological reaction conditions, at which the minimal versions were inactive (De la Pena et al., 2003; Khvorova et al., 2003). Such tertiary stabilised sequences (Figure 1C) show fast cleavage kinetics and also allow the reverse ligation reaction (Figure 1D) of hammerhead ribozymes (Canny et al., 2004; Nelson et al., 2005). Furthermore, they fold in a single step induced by magnesium ions (Penedo et al., 2004), compared to a two-step pathway shown for minimal sequences, as reviewed by Hammann and Lilley (2002). Recently, a crystal structure of the complete hammerhead ribozyme sequence from Schistosoma mansoni was obtained (Martick and Scott, 2006), which allows the reconciliation of structural and biochemical data, as reviewed in the article by W. Scott (2007) in this highlight issue.

When inspecting individual sequences of natural hammerhead ribozymes in the sub-viral RNA database (Rocheleau and Pelchat, 2006), we came across several entries that did not conform to the consensus sequence (Figure 2A). Some similar sequence variants had already been studied by Flores and co-workers, who showed that the conserved U4 in the hammerhead core can be exchanged for a $\mathrm{C}$, without affecting the in vitro and in vivo activity of the peach latent mosaic viroid (PLMVd) sequence (Ambros and Flores, 1998). We wondered whether the non-consensus database entries represented catalytically active RNA species, or rather sequencing artefacts.

To investigate the activity of these core variants (Figure $2 \mathrm{~A}$ ), we created transcripts of the PLMVd hammerhead ribozyme and initially monitored the cis-cleaving reaction during transcription (Figure 2B). Unsurprisingly, the wildtype sequence was completely cleaved within $1 \mathrm{~h}$ of transcription, and the G12A variant was inactive. Previously, the PLMVd variant G12U had failed to display any cleavage activity (Ambros et al., 1998), which is in line with the proposed role of $\mathrm{G} 12$ as a general base in the hammerhead ribozyme reaction (Han and Burke, 2005; Martick and Scott, 2006; reviewed by Przybilski and Hammann, 2006). The self-cleavage activity of the other two PLMVd variants, A9U and A13G, was slightly reduced compared to the wild-type, but was still substantial (Figure 2B). We also observed the same behaviour (data not shown) when we introduced the three core variations in the sequences of $S$. mansoni (Ferbeyre et al., 1998) and A. thaliana (Przybilski et al., 2005).

To determine the kinetic reaction parameters of the sequence variants, we created full-length cis-cleaving hammerhead ribozymes using inhibitory oligonucleotides in the transcription reactions as previously described (Przybilski et al., 2005). These RNA molecules were subjected to controlled cleavage reaction at $0.2 \mathrm{mM} \mathrm{MgCl}_{2}$. This represents a 100 -fold lower $\mathrm{Mg}^{2+}$ concentration compared to the in vitro transcriptions; however, tertiary stabilised hammerhead ribozymes retain cleavage activity under these conditions (De la Pena et al., 2003; Khvorova et al., 2003; Canny et al., 2004; Penedo et al., 2004). Furthermore, this $\mathrm{Mg}^{2+}$ concentration reduces the speed of the reaction sufficiently to measure in vitro kinetics 
A

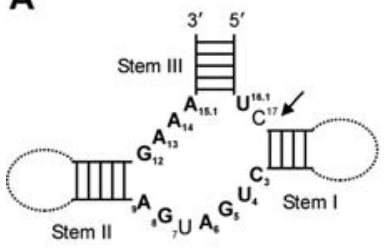

B

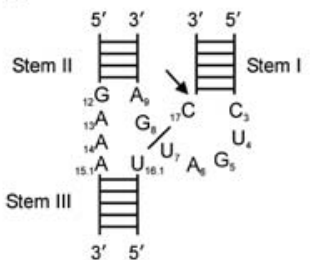

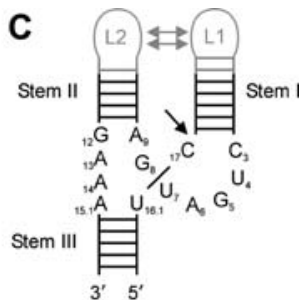

D
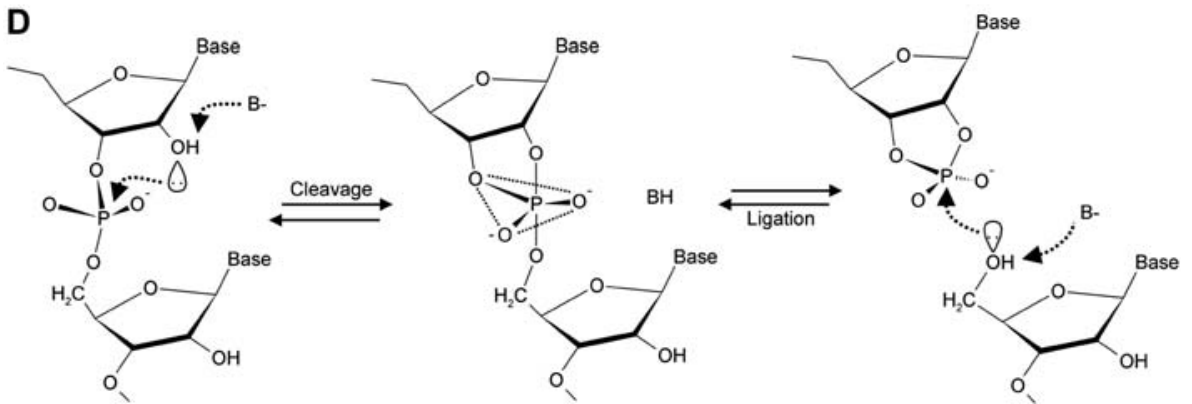

Figure 1 The hammerhead ribozyme.

(A) Secondary structure with stems I, II and III and the 11 conserved nucleotides (bold). Cleavage takes place between nucleotide 17 and the first nucleotide of stem I, as indicated by an arrow. Numbers are given according to the conventional scheme (Hertel et al., 1992). In minimal versions of the ribozyme, either stem I or stem II is closed by loops (dashed lines). Addition of divalent metal ions induces folding of the open structure depicted in $(\mathrm{A})$ and results in coaxial stacking of stems II and III in minimal versions of the hammerhead ribozyme (B) and natural ribozyme forms (C). The latter are endowed with tertiary stabilising structures formed between loops L1 and L2, which lead to activity in the sub-mM magnesium ion concentration range. (D) Transition state of the hammerhead ribozyme reaction. In the cleavage reaction, the $2^{\prime}$-oxygen of nucleotide 17 attacks the adjacent $3^{\prime}, 5^{\prime}$-phosphodiester upon removal of a hydrogen by a base (shown as B-). The trigonal-bipyramidal penta-coordinated transition state is resolved to yield a $2^{\prime}, 3^{\prime}$-cyclic phosphate and a 5'-hydroxyl group. In tertiary stabilised ribozymes $(C)$, the reverse ligation reaction is also possible.

A
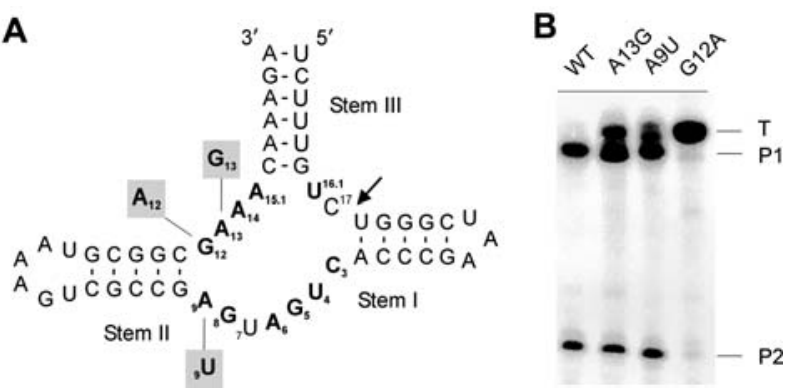

Figure 2 Sequence variants observed in viroid isolates.

(A) Isolates of the peach latent mosaic viroid (PLMVd) and the avocado sunblotch viroid (ASBVd) display the core nucleotide variations shown on the sequence of the PLMVd ribozyme. In the subviral database (http://subviral.med.uottawa.ca; Rocheleau and Pelchat, 2006) the respective entries are PLMVd.023(-) and ASBVd.061(-) for A9U, PLMVd.082(-) and ASBVd.054(+) for G12A, and PLMVd.096(-) and ASBVd.043(+) for A13G. (B) Selfcleavage of wild-type (WT) PLMVd and indicated sequence variants in the course of in vitro transcription. P1 and P2 indicate the cleavage products, and $T$ the full-length uncleaved transcript. The DNA templates for the hammerhead ribozyme sequence variants were created by recursive PCR as previously described (Przybilski et al., 2005). To ensure efficient transcription, a GGG sequence was inserted after the T7 promoter sequence. RNA was transcribed using T7 RNA polymerase (Milligan et al., 1987) in $40 \mathrm{~mm}$ Tris- $\mathrm{HCl}, \mathrm{pH}$ 8.0, $20 \mathrm{~mm} \mathrm{MgCl}$, 2 mM spermidine, $0.01 \%$ Triton X-100, with $0.5 \mathrm{~mm}$ ATP, GTP and CTP (each), $0.1 \mathrm{~mm}$ UTP and in the presence of [ $\alpha{ }^{-32}$ P]UTP. Cleavage products were resolved by PAGE on a $20 \%$ gel containing $7 \mathrm{M}$ urea and visualised by phosphorimager analysis. accurately (Canny et al., 2004). Under these reaction conditions, the three wild-type sequences of the PLMVd, A. thaliana and S. mansoni hammerhead ribozymes (Figure $3 A$ ) were active, but their first-order cleavage rate constants $\left(k_{\text {obs }}\right)$ differed by a factor of ten (Figure 3B). Since the core of these ribozymes is identical, the variations observed in the speed of the cleavage reaction must be attributable to the different sequences outside the core.

We next determined the cleavage behaviour of the A9U and $A 13 G$ variants of these ribozymes under the same reaction conditions. For the PLMVd sequence, the relative cleavage rates were $10 \%$ and $1 \%$ for A9U and A13G, respectively, compared to the wild-type sequence (inset in Figure 3B). This reduction is in the same range as that shown previously for a minimal hammerhead ribozyme (Ruffner et al., 1990). For the A. thaliana ribozyme, however, the reduction was less pronounced, while the $S$. mansoni ribozyme was least tolerant to these nucleotide exchanges (inset in Figure 3B). These data indicate that elements outside the catalytic core also determine how well changes in the core nucleotides are accepted in a given ribozyme sequence. Thus, it seems likely that the active conformation of the catalytic core is not completely identical in the different ribozymes, but rather modulated by the individual surrounding stems (Figure 3A).

In an additional set of experiments, we investigated the ligation activity (Figure 1D) of the three ribozymes and variants thereof. For this purpose, we isolated the selfcleavage products of in vitro transcription reactions and subjected them to controlled ligation reactions. The experiments were carried out under pseudo-first-order 
A
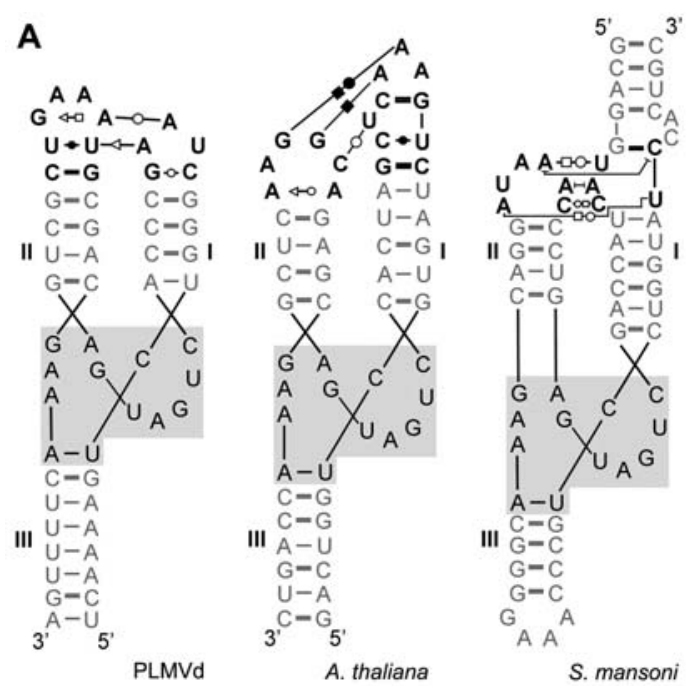

B

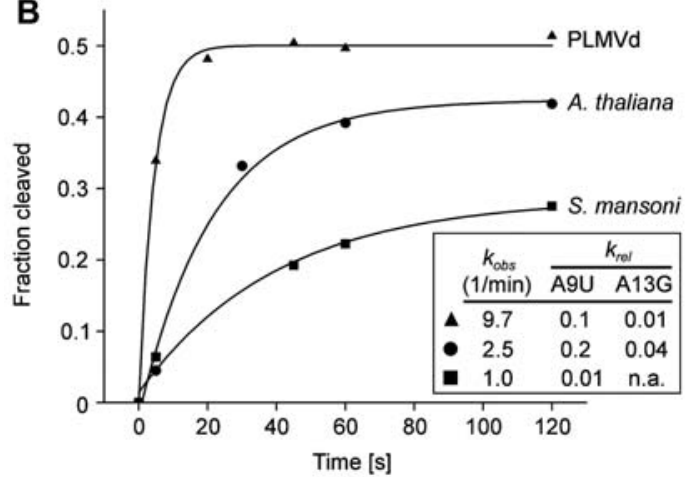

Figure 3 Cleavage activity of natural hammerhead ribozymes and core nucleotide variants.

(A) Ribozyme sequences used in this study. Helical elements are shown in grey and the conserved hammerhead ribozyme core is boxed in grey. Black lines denote backbone continuity, separated for graphical clarity. Tertiary interactions between stems I and II, inferred from modelling studies for the PLMVd and $A$. thaliana ribozymes (Khvorova et al., 2003; Przybilski et al., 2005) or crystallography for the S. mansoni sequence (Martick and Scott, 2006), are shown in black using the Leontis and Westhof (2001) annotation. (B) Progress curves of the cleavage reactions of the PLMVd $(\mathbf{\Lambda})$ A. thaliana $(\bullet)$ and S. mansoni $(\boldsymbol{\square})$ hammerhead ribozyme sequences created by plotting the cleaved fraction as a function of time. In vitro transcripts were prepared as described in the legend to Figure 2, but in the presence of the respective oligonucleotides inhibiting the ribozyme reaction. In vitro transcription reactions were resolved by PAGE as described above. Full-length transcripts were eluted from excised gel slices with $0.7 \%$ SDS $/ 40 \%$ formamide $/ 1 \times T E(10 \mathrm{mM}$ Tris-HCl, pH 8.0, 1 mM EDTA) overnight. Eluted RNA was phenolised, recovered by ethanol precipitation, and dissolved in water. RNA was snap-cooled in $20 \mathrm{~mm}$ Tris- $\mathrm{HCl}, \mathrm{pH} 7.5,0.1 \mathrm{~mm}$ EDTA, and $25 \mathrm{~mm} \mathrm{NaCl}$, and cleavage reactions at $25^{\circ} \mathrm{C}$ were started by addition of $\mathrm{MgCl}_{2}$ to an effective final concentration of $0.2 \mathrm{~mm}$. Reactions were stopped by adding 4 volumes of loading solution (95\% formamide and $50 \mathrm{~mm}$ EDTA). Cleavage products were resolved by PAGE on $20 \%$ gels containing $7 \mathrm{M}$ urea and quantified by phosphorimager analysis. Data were fitted using the equation $F(t)=F_{0}+F_{\infty}\left(1-\mathrm{e}^{-k t}\right)$ (Stage-Zimmermann and Uhlenbeck, 1998) and the resulting $k_{\text {obs }}$ values are shown in the inset. Cleavage was also monitored for the core variants A9U and $A 13 G$ of these ribozyme species and their relative rate constants $\left(k_{\text {rel }}=k_{\text {obs }}\right.$ variant $/ k_{\text {obs }}$ wT $)$ are shown in the inset. The A13G variant of $S$. mansoni was not active (n.a.). Cleavage reactions were performed at least in duplicate, and errors in $k_{\text {obs }}$ were not $>15 \%$. conditions, using traces of one radio-labelled ligation substrate in a large excess over the other, which was not labelled. The PLMVd hammerhead ribozyme showed robust ligation activity and the ligation rate constant $\left(k_{\text {obs }}\right)$ increased with $\mathrm{Mg}^{2+}$ concentration (Figure 4A). The A9U variant showed similar behaviour with slightly reduced ligation rate constants (Figure 4A). In contrast, the activity of the $A 13 G$ variant was so low that we could not determine ligation rate constants, even when tested at high $\mathrm{Mg}^{2+}$ concentrations (data not shown). In this context, it is worth noting that the ligated fraction at the end of the ligation reactions was considerably lower than the fraction that remained uncleaved in a cleavage reaction (Figure $3 \mathrm{~B}$ ). This has been observed previously and can

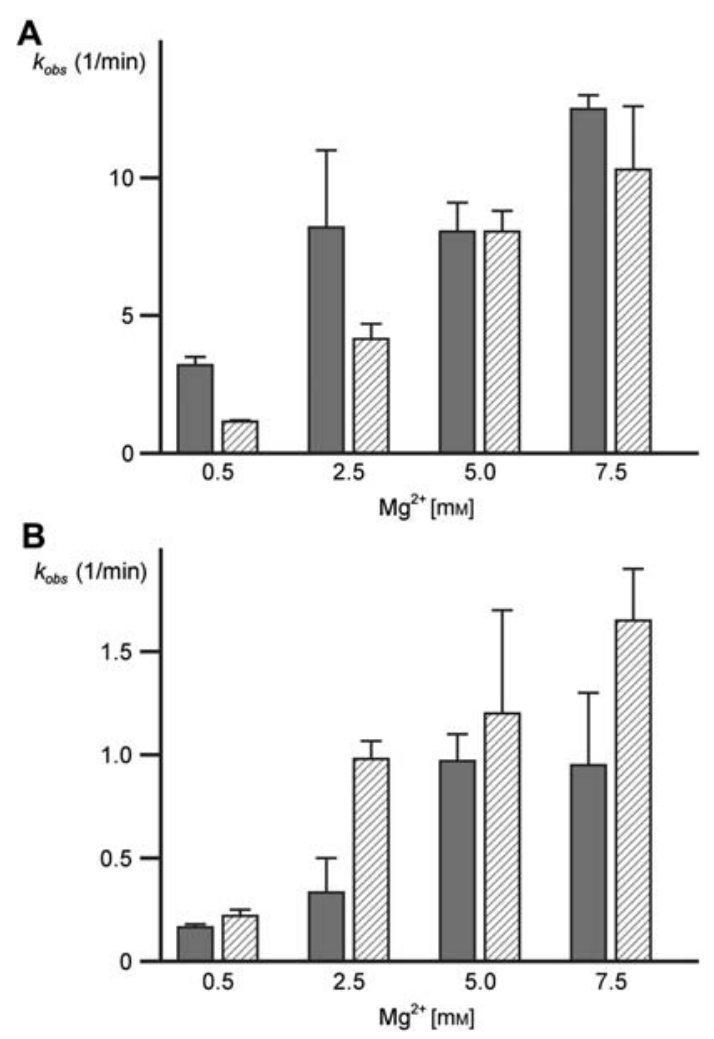

Figure 4 Ligation activity of natural hammerhead ribozymes and the A9U core variant.

Pseudo-first-order ligation rate constants are shown for reactions by the wild-type sequence (grey bars) and the A9U sequence (shaded bars) at the magnesium ion concentrations indicated for the PLMVd (A) and A. thaliana (B) ribozymes. For preparation of ligation substrates, in vitro transcriptions were carried out as described for Figure 2 or with $2.5 \mathrm{~mm}$ of each NTP. Self-cleavage products of transcriptions were gel-purified and the concentration of the non-labelled cleavage products was determined spectrophotometrically. Ligation substrates were hybridised by snap-cooling in $5 \mathrm{~mm} \mathrm{NaCl}, 10 \mathrm{~mm}$ Tris- $\mathrm{HCl}$, $\mathrm{pH} 7.5$ and $0.1 \mathrm{~mm}$ EDTA, using traces of the radio-labelled substrate and an excess of $2 \mu \mathrm{M}$ non-labelled substrate. Reactions were started by addition of $\mathrm{MgCl}_{2}$ to a final concentration of 0.5 , 2.5, 5 or $7.5 \mathrm{~mm}$. Reactions were stopped by adding 4 volumes of loading solution (95\% formamide and $50 \mathrm{~mm}$ EDTA). Ligation products were resolved by PAGE on $20 \%$ gels containing $7 \mathrm{M}$ urea and quantified by phosphorimager analysis. Data were fitted using the equation $F(t)=F_{0}+F_{\infty}\left(1-\mathrm{e}^{-k t}\right)$ (Stage-Zimmermann and Uhlenbeck, 1998) and the resulting $k_{\text {obs }}$ values are plotted. Ligation reactions were carried out at least in duplicate and the error bars denote the deviation of the mean. 
presumably be attributed to a fraction of molecules being inactive in either reaction due to the RNA purification procedures (Nelson et al., 2005). The ligation reaction seems more prone to such complications, as it requires an intact $2^{\prime}, 3^{\prime}$-cyclic phosphate at the $5^{\prime}$ ligation substrate (Figure 1D), which might survive the purification procedures to variable extents. This might also explain the greater errors in the ligation rate constants observed (Figure 4), compared to $<15 \%$ error for the cleavage rate constants.

Similar to the observations made for the PLMVd sequence, the $A$. thaliana wild-type ribozyme also displayed ligation activity (Figure 4B), while the activity of the $A 13 G$ variant was again marginal, even at a magnesium ion concentration of $7.5 \mathrm{~mm}$. The ligation reaction of the A. thaliana A9U variant, however, was consistently slightly faster than the wild-type sequence at all $\mathrm{Mg}^{2+}$ concentrations tested (Figure 4B). Surprisingly and in contrast to the sequences of PLMVd and $A$. thaliana, neither the wild-type nor the $\mathrm{A} 9 \mathrm{U}$ or $\mathrm{A} 13 \mathrm{G}$ variants of our S. mansoni ribozyme construct displayed substantial ligation activity that could be kinetically analysed. An earlier study, however, had shown ligation activity for this ribozyme, with $k_{\text {obs }}=0.32 \mathrm{~min}^{-1}$ at $1 \mathrm{mM} \mathrm{Mg}^{2+}$ (Osborne et al., 2005). Very recently, a detailed study reported efficient ligation of the $S$. mansoni ribozyme, and a lack of ligation of ASBVd and cave cricket hammerhead ribozymes (Canny et al., 2007). As discussed in that study, the fraction of active vs. inactive species in ligation constructs might be variable. At present, we cannot exclude the possibility that the low ligation activity observed for the $S$. mansoni sequence might be caused by our construct. The cleavage rate constants observed, however, are in the same range in all three studies.

The cleavage and ligation behaviour of the three hammerhead ribozymes investigated here indicate greater variability of this catalytic RNA motif than previously anticipated and the differences observed can be attributed to the individual sequences of the surrounding stems. Based on fluorescence resonance energy transfer data (Penedo et al., 2004), Lilley and co-workers have proposed a modulation of the local core structure by the tertiary interactions between stems I and II, that was later proven by the crystal structure of the S. mansoni hammerhead ribozyme (Martick and Scott, 2006). In view of the variability of the tertiary interactions in the sequences studied here (Figure 3A), it seems conceivable that they are responsible for the idiosyncratic features of the three ribozymes, implying a long-range effect mediated through stems I and II. If the local core structures were modulated in such a way, the positions of nucleotides A13 and A9 that localise in the utmost vicinity of the scissile bond (Martick and Scott, 2006) might be not identical in the three ribozymes, explaining the differences observed. The overall weak activity of the $A 13 G$ variant is likely caused by destabilisation of the transition state of the reaction (Figure 1D), as the nucleobase of position 13 is involved in hydrogen bonds to the nucleobase of C17, after which cleavage takes place. However, nucleotide A9 is also involved in stabilisation of the core structure by forming a trans Hoogsteen-sugar edge base-pair with nucleotide $\mathrm{G} 12$, which is the likely general base in the reaction (Han and Burke, 2005; Martick and Scott, 2006). A similar interaction of the A9U variant is not predicted by the isostericity matrices (Leontis et al., 2002), although this variant is apparently rather well tolerated, particularly in the $A$. thaliana ribozyme, but not when introduced in the $S$. mansoni sequence (Figure 3B). Originally, we found core variations in the sequences entered in the sub-viral RNA database (Rocheleau and Pelchat, 2006). Some of the variants, such as G12A and G12U (Ambros et al., 1998), appear to be sequencing artefacts. Although we cannot rule out that the same holds for the A13G and A9U variants, their in vitro activities under physiological reaction conditions indicate that they might be sufficiently fit for viroid processing in the cell. Next to sub-viral plant pathogens (Prody et al., 1986), hammerhead ribozymes also occur in nature in the satellite DNA of various amphibians (Epstein and Gall, 1987), schistosomes (Ferbeyre et al., 1998) and the cave cricket (Rojas et al., 2000). Most recently, they were also found in the genome of $A$. thaliana at two distinct genomic locations that are not part of a satellite DNA (Przybilski et al., 2005). In this study, we have shown that the hammerhead ribozymes from a viroid, a satellite DNA and the $A$. thaliana genome show different cleavage and ligation activities. It is conceivable that the sequence elements responsible, which surround the catalytic core, have evolved in such a way that each ribozyme can fulfil its respective biological function.

\section{Acknowledgements}

We thank Wolfgang Nellen and Gerhard Steger for helpful discussions. This work was supported by grant HA3459-3 from the Deutsche Forschungsgemeinschaft to C.H. and by the EUSTREP Fosrak. R.P. is the recipient of a stipend from the Studienstiftung des Deutschen Volkes.

\section{References}

Ambros, S. and Flores, R. (1998). In vitro and in vivo self-cleavage of a viroid RNA with a mutation in the hammerhead catalytic pocket. Nucleic Acids Res. 26, 1877-1883.

Ambros, S., Hernandez, C., Desvignes, J.C., and Flores, R. (1998). Genomic structure of three phenotypically different isolates of peach latent mosaic viroid: implications of the existence of constraints limiting the heterogeneity of viroid quasispecies. J. Virol. 72, 7397-7406.

Canny, M.D., Jucker, F.M., Kellogg, E., Khvorova, A., Jayasena, S.D., and Pardi, A. (2004). Fast cleavage kinetics of a natural hammerhead ribozyme. J. Am. Chem. Soc. 126, 1084810849.

Canny, M.D., Jucker, F.M., and Pardi, A. (2007). Efficient ligation of the Schistosoma hammerhead ribozyme. Biochemistry 46, 3826-3834.

De la Pena, M., Gago, S., and Flores, R. (2003). Peripheral regions of natural hammerhead ribozymes greatly increase their self-cleavage activity. EMBO J. 22, 5561-5570.

Epstein, L.M. and Gall, J.G. (1987). Self-cleaving transcripts of satellite DNA from the newt. Cell 48, 535-543.

Ferbeyre, G., Smith, J.M., and Cedergren, R. (1998). Schistosome satellite DNA encodes active hammerhead ribozymes. Mol. Cell. Biol. 18, 3880-3888.

Hammann, C. and Lilley, D.M. (2002). Folding and activity of the hammerhead ribozyme. ChemBioChem 3, 690-700. 
Han, J. and Burke, J.M. (2005). Model for general acid-base catalysis by the hammerhead ribozyme: $\mathrm{pH}$-activity relationships of G8 and G12 variants at the putative active site. Biochemistry 44, 7864-7870.

Hertel, K.J., Pardi, A., Uhlenbeck, O.C., Koizumi, M., Ohtsuka, E., Uesugi, S., Cedergren, R., Eckstein, F., Gerlach, W.L., Hodgson, R., and Symons, R.H. (1992). Numbering system for the hammerhead. Nucleic Acids Res. 20, 3252.

Khvorova, A., Lescoute, A., Westhof, E., and Jayasena, S.D. (2003). Sequence elements outside the hammerhead ribozyme catalytic core enable intracellular activity. Nat. Struct. Biol. 10, 708-712.

Leontis, N.B. and Westhof, E. (2001). Geometric nomenclature and classification of RNA base pairs. RNA 7, 499-512.

Leontis, N.B., Stombaugh, J., and Westhof, E. (2002). The nonWatson-Crick base pairs and their associated isostericity matrices. Nucleic Acids Res. 30, 3497-3531.

Lilley, D.M. (2005). Structure, folding and mechanisms of ribozymes. Curr. Opin. Struct. Biol. 15, 313-323.

Martick, M. and Scott, W.G. (2006). Tertiary contacts distant from the active site prime a ribozyme for catalysis. Cell 126, 309-320.

Milligan, J.F., Groebe, D.R., Witherall, G.W., and Uhlenbeck, O.C. (1987). Oligoribonucleotide synthesis using T7 RNA polymerase and synthetic DNA templates. Nucleic Acids Res. 15, 8783-8798.

Nelson, J.A., Shepotinovskaya, I., and Uhlenbeck, O.C. (2005). Hammerheads derived from sTRSV show enhanced cleavage and ligation rate constants. Biochemistry 44, 1457714585.

Osborne, E.M., Schaak, J.E., and DeRose, V.J. (2005). Characterization of a native hammerhead ribozyme derived from schistosomes. RNA 11, 187-196.
Penedo, J.C., Wilson, T.J., Jayasena, S.D., Khvorova, A., and Lilley, D.M. (2004). Folding of the natural hammerhead ribozyme is enhanced by interaction of auxiliary elements. RNA $10,880-888$.

Prody, G.A., Bakos, J.T., Buzayan, J.M., Schneider, I.R., and Bruening, G. (1986). Autolytic processing of dimeric plant virus satellite RNA. Science 231, 1577-1580.

Przybilski, R. and Hammann, C. (2006). The hammerhead ribozyme structure brought in line. ChemBioChem 7, 1641-1644.

Przybilski, R., Gräf, S., Lescoute, A., Nellen, W., Westhof, E., Steger, G., and Hammann, C. (2005). Functional hammerhead ribozymes naturally encoded in the genome of Arabidopsis thaliana. Plant Cell 17, 1877-1885.

Rocheleau, L. and Pelchat, M. (2006). The Subviral RNA Database: a toolbox for viroids, the hepatitis delta virus and satellite RNAs research. BMC Microbiol. 6, 24.

Rojas, A.A., Vazquez-Tello, A., Ferbeyre, G., Venanzetti, F., Bachmann, L., Paquin, B., Sbordoni, V., and Cedergren, R. (2000). Hammerhead-mediated processing of satellite pDo500 family transcripts from Dolichopoda cave crickets. Nucleic Acids Res. 28, 4037-4043.

Ruffner, D.E., Stormo, G.D., and Uhlenbeck, O.C. (1990). Sequence requirements of the hammerhead RNA self-cleavage reaction. Biochemistry 29, 10695-10702.

Scott, W.G. (2007). Morphing the minimal and full-length hammerhead ribozymes: implications for the cleavage mechanism. Biol. Chem. 388, 727-735.

Stage-Zimmermann, T.K. and Uhlenbeck, O.C. (1998). Hammerhead ribozyme kinetics. RNA 4, 875-889.

Uhlenbeck, O.C. (1987). A small catalytic oligoribonucleotide. Nature 328, 596-600.

Received February 13, 2007; accepted March 2, 2007 